\title{
The Treatment of Well-Differentiated Thyroid Carcinoma
}

\author{
Ralf Paschke, Thomas Lincke, Stefan P. Müller, Michael C. Kreissl, \\ Henning Dralle, Martin Fassnacht
}

\section{SUMMARY}

Background: Recent decades have seen a rise in the incidence of welldifferentiated (mainly papillary) thyroid carcinoma around the world. In Germany, the age-adjusted incidence of well-differentiated thyroid carcinoma in 2010 was 3.5 per 100000 men and 8.7 per 100000 women per year.

Method: This review is based on randomized, controlled trials and multicenter trials on the treatment of well-differentiated thyroid carcinoma that were retrieved by a selective literature search, as well as on three updated guidelines issued in the past two years.

Results: The recommended extent of surgical resection depends on whether the tumor is classified as low-risk or high-risk, so that papillary microcarcinomas, which carry a highly favorable prognosis, will not be overtreated. More than $90 \%$ of localized, well-differentiated thyroid carcinomas can be cured with a combination of surgery and radioactive iodine therapy. Radioactive iodine therapy is also effective in the treatment of well-differentiated thyroid carcinomas with distant metastases, yielding a 10-year survival rate of $90 \%$, as long as there is good iodine uptake and the tumor goes into remission after treatment; otherwise, the 10 -year survival rate is only $10 \%$. In the past two years, better treatment options have become available for radioactiveiodine-resistant thyroid carcinoma. Phase 3 studies of two different tyrosine kinase inhibitors have shown that either one can markedly prolong progression-free survival, but not overall survival. Their more common clinically significant side effects are hand-foot syndrome, hypertension, diarrhea, proteinuria, and weight loss.

Conclusion: Slow tumor growth, good resectability, and susceptibility to radioactive iodine therapy lend a favorable prognosis to most cases of welldifferentiated thyroid carcinoma. The treatment should be risk-adjusted and interdisciplinary, in accordance with the current treatment guidelines. Even metastatic thyroid carcinoma has a favorable prognosis as long as there is good iodine uptake. The newly available medical treatment options for radioactive-iodine-resistant disease need to be further studied.

\section{Cite this as:}

Paschke R, Lincke T, Müller SP, KreissI MC, Dralle H, Fassnacht M:

The treatment of well-differentiated thyroid carcinoma.

Dtsch Arztebl Int 2015; 112: 452-8. DOI: 10.3238/arztebl.2015.0452

\footnotetext{
Endocrinology and Nephrology Clinic, Leipzig University Hospital: Prof. Dr. med. Paschke

Nuclear Medicine Clinic, Leipzig University Hospital: Dr. med. Lincke

Clinic for Nuclear Medicine, University of Duisburg-Essen: Dr. med. Müller

Department of Nuclear Medicine, University Hospital of Würzburg and Klinik für Nuklearmedizin, Klinikum
} Augsburg: PD Dr. med. Kreissl

Department of General, Visceral and Vascular Surgery, University Hospital of Halle (Saale): Prof. Dr. med. Dr. h. c. Dralle

Department of Internal Medicine I, Endocrine and Diabetes Unit, University Hospital of Würzburg: Prof. Dr. Fassnacht apillary and follicular thyroid carcinoma account for $80-84 \%$ and $6-10 \%$ of all thyroid carcinomas, respectively. Both of these tumor types arise from the thyroid follicular epithelial cells; together, they are designated as well-differentiated thyroid carcinoma. They differ histologically from the rarer poorly differentiated and undifferentiated anaplastic carcinomas, which together account for $5-7 \%$ of thyroid carcinomas, as well as from medullary carcinoma of the thyroid, which arises from the parafollicular C cells and accounts for 3-5\% (1).

Well-differentiated thyroid carcinoma is the most common endocrine neoplasia, accounting for $1.2 \%$ of cancer incidence in Germany $(1.9 \%$ in women, $0.7 \%$ in men) (2). The incidence of papillary thyroid carcinoma in the USA rose by a factor of three from 1973 to 2009, with similarly marked rises around the world; in Germany, the incidence of thyroid carcinoma was about 50\% higher in 2010 than in 1998 (age-adjusted incidence in 2010: 3.5 per 100000 men and 8,7 per 100000 women per year). Small papillary thyroid carcinomas, in particular, have become much more common $(2,3)$. Most of the increased incidence is traceable to more frequent diagnostic evaluation, with improved technology (2-4). In contrast, the standardized mortality of patients with thyroid carcinoma has remained constant over the years; in Germany, in 2009 and 2010, the figure was 0.5 deaths per 100000 patients per year (2). The good prognosis of welldifferentiated thyroid carcinoma is also reflected in high 5-year survival rates: $93 \%$ for women, $88 \%$ for men. Survival is poorer for patients who are over age 45 when diagnosed and those who present with distant metastases $(2,4,5)$.

Well-differentiated thyroid carcinoma often remains clinically silent for many years, and half of all cases come to medical attention as incidental findings on physical examination or ultrasonography, or as a previously unsuspected histological finding after surgery for benign thyroid disease (4). In the systematic investigation of thyroid nodules, risk stratification is performed on the basis of the physical, ultrasonographic, and scintigraphic findings, and the diagnosis is generally established by fine-needle biopsy and cytology (6). In rarer cases, thyroid tumors present with symptoms and signs such as: 
- persistent hoarseness due to involvement of the recurrent laryngeal nerve,

- dysphagia,

- a growing nodule that is noticed by the patient,

- symptomatic cervical lymph-node metastases.

Thyroid carcinoma is generally initially classified with the "tumor-nodes-metastasis" (TI M) scheme on the basis of tumor size, infiltration of neighboring structures, lymph-node metastases, and distant metastases (Union internationale contre le cancer [UICC]/American Joint Committee on Cancer $[\mathrm{AJCC}], 7^{\text {th }}$ edition, 2009).

Prognostically relevant clinical staging takes account not only of the TI M classification, but also of the age of the patient and the histological type of the carcinoma. Most patients under age 45 have a very good prognosis and are classified as having UICC stage I disease, or stage II if they have distant metastases. The prognostically unfavorable stages III and IVA-IVC are reserved for patients aged 45 and older with primary tumors that are larger than 4 $\mathrm{cm}$ in size. Patients with undifferentiated (anaplastic) thyroid tumors of any size are generally classified as having stage IV disease, which carries a very unfavorable prognosis. In the clinical guidelines, the TI M stages are broken down into three risk groups (Table 1). Further prognostically relevant factors that are not incorporated in the TI $\mathrm{M}$ system have been identified recently, including positive BRAF mutation status (B-Raf proto-oncogene, where Raf stands for "rapidly accelerated fibrosarcoma"), which is an unfavorable molecular tumor marker in papillary thyroid carcinoma, and the depth of tissue invasion and extent of vascular invasion in follicular thyroid carcinoma $(7,8)$.

\section{Methods}

This review is based on randomized, controlled trials and multicenter therapeutic trials for well-differentiated thyroid carcinoma, which were retrieved by a selective literature search carried out in PubMed (with the searching term "thyroid carcinoma and therapy") and in the Cochrane Central Register of Controlled Trials. It also includes information from the new guideline of the Endocrine Surgery Working Group (Chirurgische Arbeitsgemeinschaft Endokrinologie, CAEK) of the German Society for General and Visceral Surgery (Deutsche Gesellschaft für Allgemein- und Viszeralchirurgie, DGAV) (9), the new guideline of the British Thyroid Association (BTA) (10), and the discussion edition of the newly updated guideline of the American Thyroid Association (ATA), along with its list of references (11).

\section{The surgical treatment of well-differentiated thyroid carcinoma}

The available evidence on surgery for welldifferentiated thyroid carcinoma is of limited value (evidence grade $\geq 2$ ). Large national registry studies $(12,13)$ and systematic reviews and meta-analyses

\section{TABLE 1}

Well-differentiated thyroid carcinoma of low, middle, and intermediate risk, as classified by the ATA and BTA (American Thyroid Association, British Thyroid Association). The ATA and BTA categories are identical, except where additional BTA criteria are noted in red (from [10, 11])

\begin{tabular}{|c|c|c|}
\hline Risk & pTN & Further features \\
\hline Low & $\begin{array}{l}\text { pT1a solitary or multiple } \\
\text { NO/Nx } \\
\text { pT1b No/Nx } \\
\text { pT2 NO/Nx } \\
\text { pT3 }>4 \mathrm{~cm} \mathrm{N0/Nx}\end{array}$ & $\begin{array}{l}<4 \mathrm{~cm} \text { intrathyroid } \\
\text { R0, R1 } \\
\text { M0 }\end{array}$ \\
\hline Middle & $\begin{array}{l}\text { pT3 N0 Nx } \\
\text { pT3 N1a or N1b }\end{array}$ & $\begin{array}{l}\text { in case of spread beyond the thyroid: } \\
\text { microscopic } \\
\text { R0 } \\
\text { M0 }\end{array}$ \\
\hline High & $\begin{array}{l}\text { pT4 } \\
\text { M1 } \\
\text { R2 }\end{array}$ & \\
\hline
\end{tabular}

are available (14), but no randomized, controlled trials (RCTs) have yet been performed (15).

The histological type of carcinoma and the extent of local and regional spread determine the recommended extent of primary surgical resection of papillary and follicular thyroid carcinoma. In contrast, secondary factors that could affect the individual prognosis, such as the age of the patient or the molecular status of the tumor (e.g., a BRAF mutation in a papillary tumor), play no role in the current guidelines' recommendations for the surgical treatment of well-differentiated thyroid carcinoma (9, 11).

Recent years have seen a marked increase in the incidental discovery of papillary microcarcinomas (i.e., carcinomas no greater than $10 \mathrm{~mm}$ in size), which have a low risk of recurrence or metastasis. It has, therefore, become necessary to distinguish lowrisk from high-risk tumors in surgical treatment planning, so that low-risk tumors will not be subjected to overly aggressive resection, which would cause more complications without improving oncologic outcomes (Table 2) (12). I on-metastatic papillary microcarcinomas and minimally invasive follicular carcinomas without vascular invasion are low-risk tumors; papillary tumors over $10 \mathrm{~mm}$ in size (regardless of their primary metastatic status) and follicular tumors that show vascular invasion or are widely invasive are high-risk tumors (Table 2). Intrathyroid papillary tumors without any clinically demonstrable lymph node metastases (cI 0), multifocal papillary microcarcinomas, and minimally invasive oncocytic follicular tumors (Hürthle-cell tumors) seem to occupy an intermediate position between low- and high-risk tumors, and the extent of resection can be planned accordingly; this matter is not yet fully clear. There is, however, an international consensus that extensive resections should be carried out in centers with special expertise to 
TABLE 2

Recommended extent of resection for papillary and follicular thyroid carcinoma with low, increased, and high risk (summarized from [9])

\begin{tabular}{|c|c|c|c|c|}
\hline $\begin{array}{l}\text { Risk } \\
\text { category }\end{array}$ & $\begin{array}{l}\text { Type of } \\
\text { carcinoma }\end{array}$ & Degree of spread & Recommended extent of resection & Alternative \\
\hline \multirow{2}{*}{ Low } & papillary & $\begin{array}{l}\leq 10 \mathrm{~mm} \text {, solitary, no extrathyroid invasion, no } \\
\text { lymph-node metastases or distant metastases }\end{array}$ & hemithyroidectomy & $\begin{array}{l}\text { total thyroidectomy if there is a } \\
\text { contralateral nodule }\end{array}$ \\
\hline & follicular & $\begin{array}{l}\text { minimally invasive without vascular invasion } \\
\text { or metastases }\end{array}$ & hemithyroidectomy & $\begin{array}{l}\text { total thyroidectomy if there is a } \\
\text { contralateral nodule }\end{array}$ \\
\hline \multirow[b]{2}{*}{$\begin{array}{l}\text { Increased } \\
\text { or high }\end{array}$} & papillary & $\begin{array}{l}>10 \mathrm{~mm} \text {, multifocal or with extrathyroid } \\
\text { invasion, lymph-node metastases, } \\
\text { or distant metastases }\end{array}$ & $\begin{array}{l}\text { total thyroidectomy with central lymph-node } \\
\text { dissection; lateral lymph-node dissection } \\
\text { depending on tumor involvement }\end{array}$ & $\begin{array}{l}\text { total thyroidectomy without } \\
\text { lymph-node dissection in the } \\
\text { cNOcM0 situation }\end{array}$ \\
\hline & follicular & $\begin{array}{l}\text { minimally invasive with vascular invasion, } \\
\text { or widely invasive }\end{array}$ & $\begin{array}{l}\text { total thyroidectomy without prophylactic } \\
\text { lymph-node dissection }\end{array}$ & $\begin{array}{l}\text { total thyroidectomy and } \\
\text { prophylactic lymph-node } \\
\text { dissection in case of oncocytic } \\
\text { follicular carcinoma of the } \\
\text { thyroid }\end{array}$ \\
\hline
\end{tabular}

limit the risk of complications, especially postoperative hypoparathyroidism and hypocalcemia $(9,11$, 13).

The classification of the local and regional lymph node system and the concept of lymph node surgery in well-differentiated thyroid carcinoma have met with important refinements in recent years, based on a multitude of studies (16). Prophylactic lymph-node dissection has been found to provide no significant advantage with respect to the risk of local and regional recurrence if there is no clinical or radiological evidence of lymph-node metastases. If the lymph nodes are clinically or radiologically positive, a compartment-oriented lymph-node dissection can lower the rates of recurrence and reoperation (9).

More than $90 \%$ of localized, well-differentiated carcinomas of the thyroid can now be cured by surgery combined with radioactive iodine therapy (9). Resectability is limited mainly by infiltration of the respiratory or gastrointestinal tracts in the mediastinum. For tumor invasion confined to the neck, various resective techniques have been devised, depending on the site and the longitudinal and transverse extent of the tumor (e.g., so-called fenestration or segmental resection of the trachea). The use of these techniques enables the potentially curative local resection of locally advanced welldifferentiated tumors and is justified by higher survival rates $(9,16)$.

\section{Radioactive iodine therapy}

The second pillar of treatment for well-differentiated thyroid carcinoma, after surgery, is radioactive iodine therapy. In this context, "radioactive iodine" refers to the isotope ${ }^{131} \mathrm{I}$, which emits beta radiation with a mean tissue penetration of ca. $1 \mathrm{~mm}$, as well as a more deeply penetrating gamma radiation that can be used for scintigraphy. Well-differentiated thyroid carcinomas generally retain the ability to take up and fix iodine when stimulated by thyroid- stimulating hormone (TSH) at concentrations above $30 \mathrm{mU} / \mathrm{L}$; when radioactive iodine is administered, the tumor receives a high radiation dose, while the surrounding normal tissue is largely spared. Thus, even without precise localization of the tumor and its potential spread, radiotherapy can be delivered specifically to the cancerous tissue $(17,18)$.

Radioactive iodine therapy is a standard treatment in Germany despite the absence of randomized, controlled trials. Clinical trials to date have yielded inconsistent results, and the evidence base for radioactive iodine therapy remains limited (evidence grade $\geq 2$ ), even in the most recent guidelines (18). The favorable prognosis of well-differentiated thyroid carcinoma makes robust data hard to obtain. In all current guidelines, radioactive iodine therapy is said to be clearly indicated for the treatment of metastases that take up iodine and are not amenable to curative resection $(10,11,17)$; such metastases usually arise in the lungs and in bone. The guidelines vary in their recommendations about adjuvant radioactive iodine therapy for low-risk thyroid carcinomas (18).

The German guideline (17), which is now being updated, contains a general recommendation for adjuvant radioactive iodine therapy at a dose of 2.0-3.7 GBq ${ }^{131} \mathrm{I}$. A more restrictive recommendation is given solely for unifocal papillary microcarcinoma (up to $1 \mathrm{~cm}$ in size): in such cases, adjuvant radioactive iodine therapy should be given only if unfavorable prognostic factors are present.

The ATA guidelines of 2009 (11) and the BTA guidelines of 2014 (10) restrict the absolute indication for adjuvant radioactive iodine therapy to tumors larger than $4 \mathrm{~cm}$ and those that macroscopically infiltrate the cervical soft tissues or have known distant metastases. In all other cases, the indication for adjuvant radioactive iodine therapy depends on individual risk factors:

- known lymph node metastases

- age 


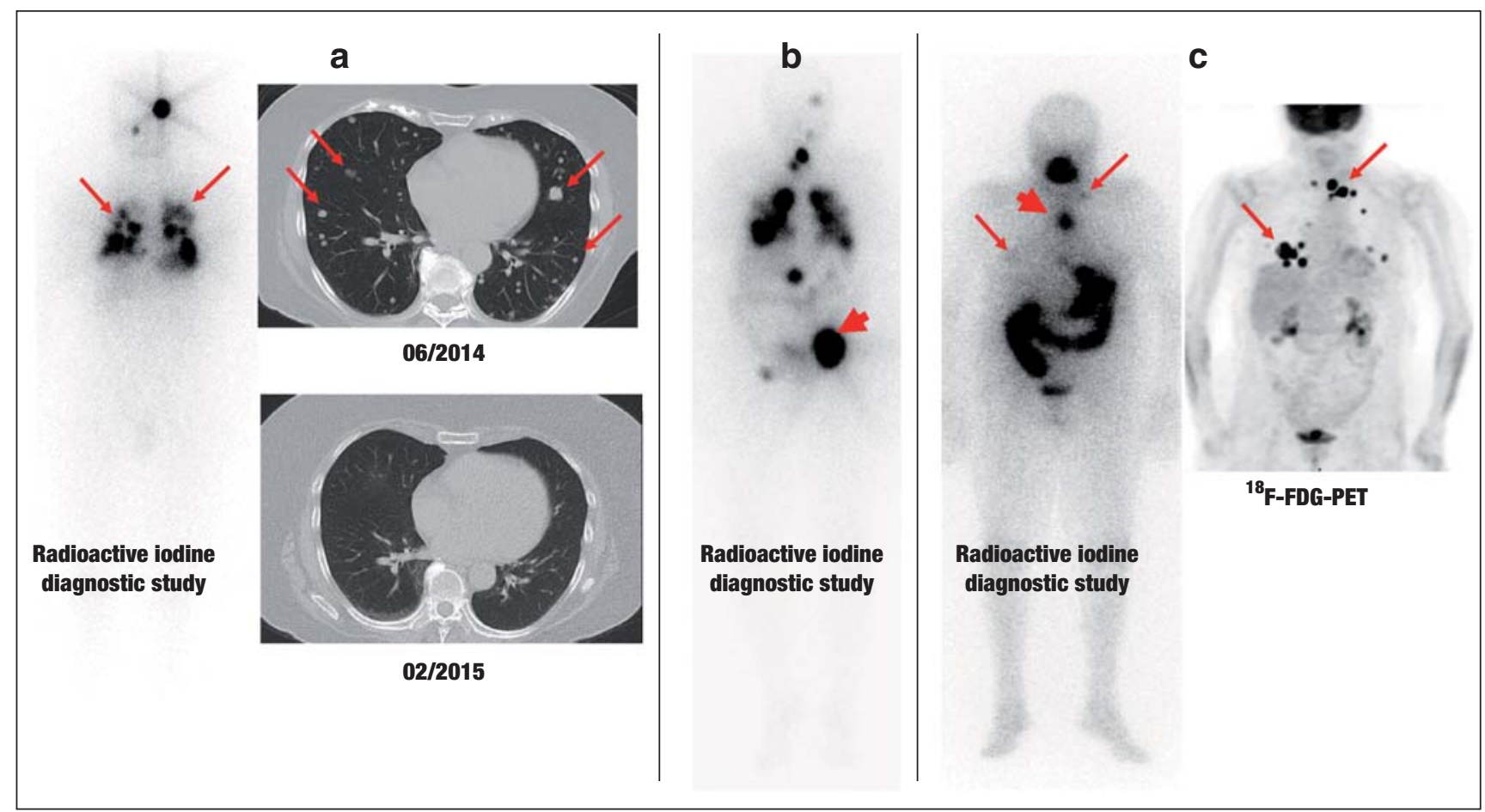

Figure: Three illustrative cases of patients with metastatic thyroid carcinoma.

a) Follicular thyroid carcinoma with very good radioactive iodine uptake (left). One year after radioactive iodine therapy, CT reveals only slight evidence of possible residual tumor at the site of the original pulmonary metastases (arrows).

b) Follicular thyroid carcinoma with metastases to bone, lungs, and mediastinum. Scintigraphy after radioactive iodine therapy reveals marked radioactive iodine uptake in all of the metastases. A large, osteolytic metastasis to the pelvis (arrow) was additionally treated with external beam radiotherapy, because, despite marked uptake of radioactive iodine, this form of treatment alone die not yield a sufficient radiation dose.

c) Poorly differentiated thyroid carcinoma. The whole-body radioactive iodine diagnostic study (left) reveals a mediastinal lymph-node metastasis with marked uptake (thick arrow), but without any correlate in the FDG-PET (right). In contrast, the supraclavicular lymph nodes take up large amounts of FDG, but hardly any radioactive iodine (thin arrows).

Standard imaging studies in thyroid carcinoma include ultrasonography of the neck and radioactive iodine scintigraphy (once or twice), and, in some advanced cases, tomographic imaging and FDG-PET.

CT, computerized tomography; FDG, fluorodeoxyglucose; FDG-PET, fluorodeoxyglucose positron emission tomography.

- tumor size

- invasiveness

- aggressive histologic subtypes.

If no risk factors are present, radioactive iodine therapy is not recommended. In cases with low risk, the ATA and BTA recommend treatment with 1.1-3.7 GBq ${ }^{131} \mathrm{I}$ and $1.1 \mathrm{GBq}{ }^{131} \mathrm{I}$, respectively. In cases with increased risk, the ATA recommends treatment with 3.7-7.4 GBq ${ }^{131} \mathrm{I}$, while the BTA recommends individualized dose escalation.

Radioactive iodine therapy is given on an adjuvant basis after thyroidectomy to ablate residual thyroid tissue, after total thyroidectomy to eliminate potential occult metastasis, and to facilitate postoperative care with the measurement of thyroglobulin levels. Wholebody scintigraphy three days or more after radioactive iodine application completes the initial staging. Radioactive iodine therapy is also the most effective treatment for metastases that take up radioactive iodine and cannot be curatively resected. It yields a 10 -year survival rate of $90 \%$ among patients who have welldifferentiated thyroid carcinomas with distant metastases and with good iodine uptake, if they have a complete remission after their first radioactive iodine treatment; patients who have distant metastases without any initial radioactive iodine uptake have a 10 -year survival rate of only $10 \%(17,20)$.

A sufficient level of TSH stimulation for radioactive iodine therapy is usually reached 3-4 weeks after thyroidectomy without postoperative thyroid hormone substitution, or 3-4 weeks after the discontinuation of levothyroxine administration (liothyronine can be given for bridging treatment). Alternatively, a recombinant human TSH preparation has been approved for use in adjuvant radioactive iodine therapy and obviates the need to discontinue levothyroxine and induce transient hypothyroidism $(10,11)$.

For post-treatment follow-up, the German guidelines contain a recommendation for whole-body scintigraphy with radioactive iodine $(100-400 \mathrm{MBq})$ or ${ }^{123} \mathrm{I}$ (40-200 MBq), under stimulation with endogenous or exogenous TSH, 3-6 months after radioactive iodine therapy to confirm successful tumor ablation. This can be dispensed with in patients at low risk: 
tumor size less than $4 \mathrm{~cm}$

- no lymph-node involvement

- negative post-treatment scintigraphy

- tumor marker thyroglobulin $<2 \mathrm{ng} / \mathrm{mL}$.

The ATA and BTA likewise see no indication for whole-body scintigraphy in low-risk patients $(10,11)$.

\section{Radioactive iodine resistance}

As discussed above, the prognosis is markedly worse if the tumor does not take up radioactive iodine, because radioactive iodine therapy to prevent (or treat) recurrent disease - which is otherwise indicated in most clinical situations - is then of no use. The literature provides no uniform definition of "radioactive iodine resistance" in patients with well-differentiated thyroid carcinoma. Very recently, a panel of experts considered the definition and management of this entity (19). Generally speaking, radioactive iodine resistance is said to be present if the primary tumor or its metastases do not take up radioactive iodine, either initially or in their further course, or if a well-conducted course of radioactive iodine therapy does not result in the generation of a clinically useful radiation dose in tumor tissue. Further criteria include tumor persistence despite a cumulative therapeutic activity of $22 \mathrm{GBq}$ or more (20) and tumor progression within 12-18 months of the receipt of a therapeutic dose of radioactive iodine.

There has been major recent progress in the biological characterization of radioactive iodine resistance. The BRAF-V600E mutation (a mutation of the BRAF gene in codon V600 to E600, with a frequency of ca. $60 \%$ in papillary thyroid carcinoma) plays an important role in radioactive iodine resistance in papillary and poorly differentiated thyroid carcinoma (21-23). Tumors displaying this mutation take a more aggressive course and store radioactive iodine to a markedly lesser extent, even though they are often positive in fluorodeoxyglucose positron emission tomography (FDG-PET) (Figure). In contrast, it seems that tumors with radio-iodine-positive metastases more often display a rat sarcoma proto-oncogene (RAS) mutation (24). Data on the frequency of radioactive iodine resistance are highly variable, as the findings depend to a large extent on the group of patients studied and on the particular diagnostic tests that are carried out. In the largest series on metastatic well-differentiated thyroid carcinoma, published in 2006, the tumors failed to take up radioactive iodine in 132 of 427 patients $(31 \%)$, and a further 168 patients $(39 \%)$ did not go into remission after treatment despite the presence of radioactive iodine uptake (20). The 10-year survival rate among patients with uptake-negative tumors was only $10 \%$, while that among patients with uptake-positive tumors was $90 \%$ if an initial remission was achieved and $29 \%$ if it was not (20).

\section{The pharmacotherapy of thyroid carcinoma}

The growth of thyroid cells depends on TSH, and TSHsuppressive thyroxine administration is a cornerstone of the further care of patients with well-differentiated thyroid carcinoma after surgery and radioactive iodine therapy (11). The desired extent of TSH suppression depends on the risk classification (Table 1) (11). Persistent postoperative hypoparathyroidism is treated with active vitamin D3 hormone and calcium. Both treatments require regular follow-up and adjustment when indicated (9-11).

For radioactive-iodine-resistant thyroid carcinoma, data have recently become available from two placebocontrolled phase III trials (evidence grade Ib) $(25,26)$. The multitargeted tyrosine kinase inhibitors sorafenib and lenvatinib are directed against the VEGF (vascular endothelial growth factor) receptor family, and lenvatinib additionally inhibits FGF (fibroblast growth factor) receptors. I o significant overall survival advantage has been demonstrated for patients taking either of these tyrosine kinase inhibitors. $64 \%$ of patients taking sorafenib had to reduce the dose from the initial value of $400 \mathrm{mg}$ b.i.d.; $19 \%$ had to discontinue the treatment because of side effects. The more common major side effects were:

- hand-foot syndrome $(20.3 \%)$

- hypertension $(9.7 \%)$

- diarrhea $(5.8 \%)$

- weight loss $(5.8 \%)$

- fatigue $(5.8 \%)$.

Lenvatinib treatment had to be interrupted at least temporarily in $82 \%$ of patients, and discontinued in $14 \%$. The more common major side effects were $(25,26)$ :

- hypertension $(41.8 \%)$

- proteinuria $(10 \%)$

- fatigue $(9.2 \%)$

- weight loss $(9.6 \%)$

- diarrhea $(8.0 \%)$.

6 of the 20 deaths among patients taking lenvatinib (rather than placebo) were ascribed to the drug.

In a trial involving 417 patients with progressive, radioactive-iodine-resistant thyroid carcinoma, sorafenib significantly prolonged median progression-free survival (10.8 vs 5.8 months; hazard ratio [HR] 0.59; $<<0.0001$ ) (25). A partial remission (objective tumor response) was seen in $12.2 \%$ of the patients taking sorafenib and $0.5 \%$ of those taking placebo. The median duration of remission under treatment with sorafenib was 10.2 months, but there were no complete remissions. These favorable results, in comparison to those of conventional chemotherapy, led to the approval of sorafenib in April 2014 by the European Medicines Agency for the treatment of progressive, radioactive-iodine-resistant thyroid carcinoma. The daily cost of long-term treatment with this drug is $€ 174$ as of May 2015.

In another placebo-controlled trial involving 392 patients, lenvatinib prolonged median progression-free survival from 3.6 to 18.3 months (HR $0.21 ; \mathrm{p}<0.0001$ ) (25) and brought about an objective tumor response in $65 \%$ of patients, as opposed to only $1.5 \%$ with placebo. Four patients taking lenvatinib had a complete remission. I otably, the results in a subgroup of 93 patients who had been previously treated with anti-VEGF therapy were just as good as those in patients receiving first-line treatment. Lenvatinib has not yet been approved for use in Europe. 
Although these data might seem to imply that lenvatinib is somewhat more effective than sorafenib, it must be pointed out that the placebo groups in the two studies differed in their median progression-free survival (5.6 vs. 3.6 months). The lack of demonstration of a significant overall survival benefit for either drug, the need for long-term treatment, and the side effects mentioned above-some of which markedly impair patients' quality of life — are all reasons why these drugs should only be given to carefully selected patients in a specialized, interdisciplinary center for the treatment of thyroid carcinoma. Other treatments (surgery, radiotherapy) should generally be tried first. Tyrosine kinase inhibitors should be given only if the following criteria are met:

- unresectable tumors

- clinically significant tumor mass

- definite radioactive iodine resistance

- documented progression (this may mean that a period of observation is needed before treatment)

- the patient must be informed of the potential side effects and of the lack of evidence for a significant benefit with respect to overall survival

- the patient must have a strong desire for treatment.

Treatment with these drugs must be supervised by physicians who are experienced in dealing with the side effects of tyrosine kinase inhibitors and in managing their rare serious complications (25-27). The prevention of imminent death from tumor progression can be a valid reason for treatment. The indications for treatment with tyrosine kinase inhibitors (19) are currently undergoing further development through exploratory analysis of the two approval studies and experience gained from the use of the drugs in practice. Thus, it is still too early to state what percentage of patients will ultimately be amenable to treatment with these drugs.

\footnotetext{
Conflict of interest statement

Prof. Paschke has served as a paid consultant for Bayer and Eisai and has received reimbursement of meeting participation fees and of travel and accommodation expenses from these two companies. He has received thirdparty funding from Bayer, Eisai, and AstraZeneca for performing clinical trials on behalf of these companies.

Dr. Lincke has received reimbursement of meeting participation fees and of travel and accommodation expenses from Bayer Health Care.

Dr. Müller has been paid for serving on advisory boards for Exelixis/SOBI and Bayer.

PD Dr. Kreissl has served as a paid consultant for AstraZeneca, Eisai, Bayer, and $\mathrm{SOBI}$. He has received reimbursement of meeting participation fees and of travel and accommodation expenses from AstraZeneca and SOBI. He has received payment for preparing scientific meeting programs from Genzyme, Bayer, and AstraZeneca. He has received third-party funding from Bayer, Eisai,Genzyme, and AstraZeneca for performing clinical trials on behalf of these companies.

Prof. Fassnacht has served as a paid consultant for Bayer, Eisai, AstraZeneca and $\mathrm{SOBI}$ and has received a lecture honorarium from Eisai. He has received third-party funding from Bayer for carrying out a clinical trial.

Prof. Dralle states that he has no conflict of interest.
}

Manuscript submitted on 22 0ctober 2014, revised version accepted on 8 April 2015.

Translated from the original German by Ethan Taub, M.D.
KEY MESSAGES

- The treatment of well-differentiated thyroid carcinoma should be risk-adjusted and interdisciplinary, in accordance with the current treatment guidelines.

- Because of the marked increase in the incidental discovery of papillary microcarcinomas in recent years, the distinction between so-called low- and high-risk tumors is an important factor in therapeutic decisionmaking.

- Surgery and radioactive iodine therapy are the first and second pillars of treatment for well-differentiated thyroid carcinoma.

- Thyroid carcinoma is said to be resistant to radioactive iodine therapy if the primary tumor or its metastases do not take up radioactive iodine, either initially or in their further course, or if a well-conducted course of radioactive iodine therapy does not result in the generation of a clinically useful radiation dose in tumor tissue.

- In the past two years, better treatment options have become available for radioactive-iodine-resistant thyroid carcinoma. Phase 3 studies of two tyrosine kinase inhibitors have shown that either one can markedly prolong progression-free survival, but not overall survival. One of these drugs has been approved for use in Germany.

\section{REFERENCES}

1. Ceresini G, Corcione L, Michiara M, et al.: Thyroid cancer incidence by histological type and related variants in a mildly iodine-deficient area of Northern Italy, 1998 to 2009. Cancer 2012; 118: 5473-80.

2. Robert Koch-Institut und Gesellschaft der epidemiologischen Krebsregister in Deutschland e. V. (eds.): Krebs in Deutschland 2009/2010. 9. Ausgabe. Berlin, 2013.

3. Morris LGT, Sikora AG, Tosteson TD, Davies L: The increasing incidence of thyroid cancer: The influence of access to care. Thyroid 2013; 23: 885-91.

4. Tuttle RM, Ball DW, Byrd D, et al.: Thyroid carcinoma. J Natl Compr Canc Netw 2010; 8: 1228-74.

5. Agate L, Lorusso L, Elisei R: New and old knowledge on differentiated thyroid cancer epidemiology and risk factors. J Endocrinol Invest 2012; 35: 3-9.

6. Gharib H, Papini E, Paschke R, et al. for the AACE/AME/ETA Task Force on Thyroid Nodules: American Association of Clinical Endocrinologists, Associazione Medici Endocrinologi and European Thyroid Association: medical guidelines for clinical practice for the diagnosis and management of thyroid nodules. Endocr Pract 2010; 16: 1-43.

7. Nikiforov Y: Molecular diagnostics of thyroid tumors. Arch Patho Lab Med 2011; 135: 569-77

8. Hermann M, Tonninger K, Kober F, Furtlehner EM, Schultheis A, Neuhold N: Minimal-invasives follikuläres Schilddrüsenkarzinom. Eine Thyreoidektomie ist nicht obligat. Chirurg 2010; 81: 627-35.

9. Dralle H, Musholt TJ, Schabram J, et al.: German Association of Endocrine Surgeons practice guideline for the surgical management of malignant thyroid tumors. Langenbecks Arch Surg 2013; 398: 347-75.

10. Perros P, Colley S, Boelaert K, et al.: Guidelines for the management of thyroid cancer. Clin Endocrinol 2014; 81: 1-122. 
11. Cooper DS, Doherty GM, Haugen BR, et al.: Revised American Thyroid Association management guidelines for patients with thyroid nodules and differentiated thyroid cancer. Thyroid 2009; 19 : 1167-214.

12. Adam MA, Pura J, Gu L, et al.: Extent of surgery for papillary thyroid cancer is not associated with survival. Ann Surg 2014; 260:

601-7.

13. Bilimoria KY, Bentrem DJ, Ko CY, et al.: Extent of surgery affects survival for papillary thyroid cancer. Ann Surg 2007; 246: 375-81.

14. Lang BH, Ng SH, Lau L, Cowling B, Wong KP, Wan KY: A systematic review and meta-analysis of prophylactic central neck dissection on short-term locoregional recurrence in papillary thyroid carcinoma after total thyroidectomy. Thyroid 2013; 23: 1087-98.

15. Carling T, Carty SE, Ciarleglio MM, et al.: American Thyroid Association design and feasibility of a prospective randomized controlled trial of prophylactic central lymph node dissection for papillary thyroid carcinoma. Thyroid 2012; 22: 237-44.

16. Lorenz K, Niederle B, Steinmüller T, Dralle H: The European Society of Endocrine Surgeons perspective of thyroid cancer surgery: an evidence-based approach. Langenbecks Arch Surg 2014; 399 135-9.

17. Dietlein M, Dressler J, Eschner W, et al.: Procedure guidelines for radioiodine therapy of differentiated thyroid cancer (version 3). Nuklearmedizin 2007; 46: 213-19.

18. Dietlein M, Verburg FA, Luster M, et al.: One should not just read what one believes: the nearly irresolvable issue of producing truly objective, evidence-based guidelines for the management of diffrentiated thyroid cancer. Eur J Nucl Med Mol Imaging 2011; 38: 793-8.

19. Schlumberger M, Brose M, Elisei R, et al.: Definition and management of radioactive iodine-refractory differentiated thyroid cancer. Lancet Diabetes Endocrinol 2014; 2: 356-8.

20. Durante C, Haddy N, Baudin E, et al.: Long-term outcome of 444 patients with distant metastases from papillary and follicular thyroid carcinoma: benefits and limits of radioiodine therapy. J Clin Endocrinol Metab 2006; 91: 2892-9.

21. Elisei R, Ugolini C, Viola D, et al.: BRAF(V600E) mutation and outcome of patients with papillary thyroid carcinoma: a 15-year median follow-up study. J Clin Endocrinol 2008; 93: 3943-9.

22. Ricarte-Filho JC, Ryder M, Chitale DA, et al.: Mutational profile of advanced primary and metastatic radioactive iodine-refractory thyroid cancers reveals distinct pathogenetic roles for BRAF, PIK3CA, and AKT1. Cancer Res 2009; 69: 4885-93.

23. Yang $\mathrm{K}$, Wang $\mathrm{H}$, Liang Z, Liang J, Li F, Lin Y: BRAFV600E mutation associated with non-radioiodine-avid status in distant metastatic papillary thyroid carcinoma. Clini Nucl Mmed 2014; 39: 675-9.

24. Sabra MM, Dominguez JM, Grewal RK, et al.: Clinical outcomes and molecular profile of differentiated thyroid cancers with radioiodineavid distant metastases. J Clin Endocrinol 2013; 98: E829-36.

25. Brose MS, Nutting CM, Jarzab B, et al.: Sorafenib in radioactive iodine-refractory, locally advanced or metastatic differentiated thyroid cancer: a randomised, double-blind, phase 3 trial. Lancet 2014; 384: 319-28.

26. Schlumberger M, Tahara M, Wirth LJ, et al.: Lenvatinib versus placebo in radioiodine refractory thyroid cancer. N Engl J Med 2015; 372: 621-30.

27. Blevins DP, Dadu R, Hu M, et al.: Aerodigestive fistula formation as rare side effect of antiangiogenic tyrosine kinase inhibitor therapy for thyroid cancer. Thyroid 2014; 31: 918-22.

\section{Corresponding autho}

Prof. Dr. Ralf Paschke

Klinik für Endokrinologie und Nephrologie

Universitätsklinikum Leipzig

Liebigstr. 20, D-04103 Leipzig, Germany

ralf.paschke@medizin.uni-leipzig.de 